\title{
A tip/tilt mirror with large dynamic range for the ESO VLT Four Laser Guide Star Facility
}

\author{
N. Rijnveld ${ }^{\mathrm{a}}$, R. Henselmans ${ }^{\mathrm{a}}$, B. Nijland ${ }^{\mathrm{a}}$ \\ ${ }^{a}$ TNO, Optomechatronics, Stieltjesweg 1, 2628 CK Delft, The Netherlands
}

\begin{abstract}
One of the critical elements in the Four Laser Guide Star Facility (4LGSF) for the ESO Very Large Telescope (VLT) is the Optical Tube Assembly (OTA), consisting of a stable 20x laser beam expander and an active tip/tilt mirror, the Field Selector Mechanism (FSM). This paper describes the design and performance testing of the FSM. The driving requirement for the FSM is its large stroke of $\pm 6.1 \mathrm{mrad}$, in combination with less than $1.5 \mu \mathrm{rad}$ RMS absolute accuracy. The FSM design consists of a Zerodur mirror, bonded to a membrane spring and strut combination to allow only tip and tilt. Two spindle drives actuate the mirror, using a stiffness based transmission to increase resolution. Absolute accuracy is achieved with two differential inductive sensor pairs. A prototype of the FSM is realized to optimize the control configuration and measure its performance. Friction in the spindle drive is overcome by creating a local velocity control loop between the spindle drives and the shaft encoders. Accuracy is achieved by using a cascaded low bandwidth control loop with feedback from the inductive sensors. The pointing jitter and settling time of the FSM are measured with an autocollimator. The system performance meets the strict requirements, and is ready to be implemented in the first OTA.
\end{abstract}

Keywords: VLT, Adaptive Optics, Laser Guide Star, OTA, Field Selector, tip/tilt mirror, mechatronics

\section{INTRODUCTION}

ESO is implementing an Adaptive Optics facility (AOF) on Unit Telescope 4 (UT4) of the Very Large Telescopes (VLT) [1], see Figure 1. For increased sky coverage, four Laser Guide Star Facilities (4LGSF) [2][3] will be installed. A critical element in the 4LGSF is the Optical Tube Assembly (OTA). Each OTA consists of a 20x laser beam expander to create a stable collimated laser beam of $\varnothing 300 \mathrm{~mm}$, and a tip/tilt beam steering mechanism to cover an on-sky field of view of radius 4.8 arc min. The tip/tilt mechanism is called the Field Selector Mechanism (FSM), and is the subject of this paper. This paper describes the design and performance testing of the first prototype of the FSM, as part of the TNO project to design and realize the four OTAs. The driving requirement for the FSM is its large mechanical stroke of $\pm 6.1 \mathrm{mrad}$ combined with less than $1.5 \mu \mathrm{rad}$ RMS absolute accuracy, and its step response: $21.2 \mu \mathrm{rad}$ in tip direction and $15.0 \mu \mathrm{rad}$ in tilt direction, both in less than 0.2 seconds. Note that the absolute accuracy requirement contains dynamic requirements on the pointing jitter of the mirror, as well as on the absolute pointing knowledge with respect to a fixed reference. This paper focuses on the pointing jitter and accuracy of the closed loop system, and does not report the temperature and calibration tests performed to achieve the required absolute accuracy.

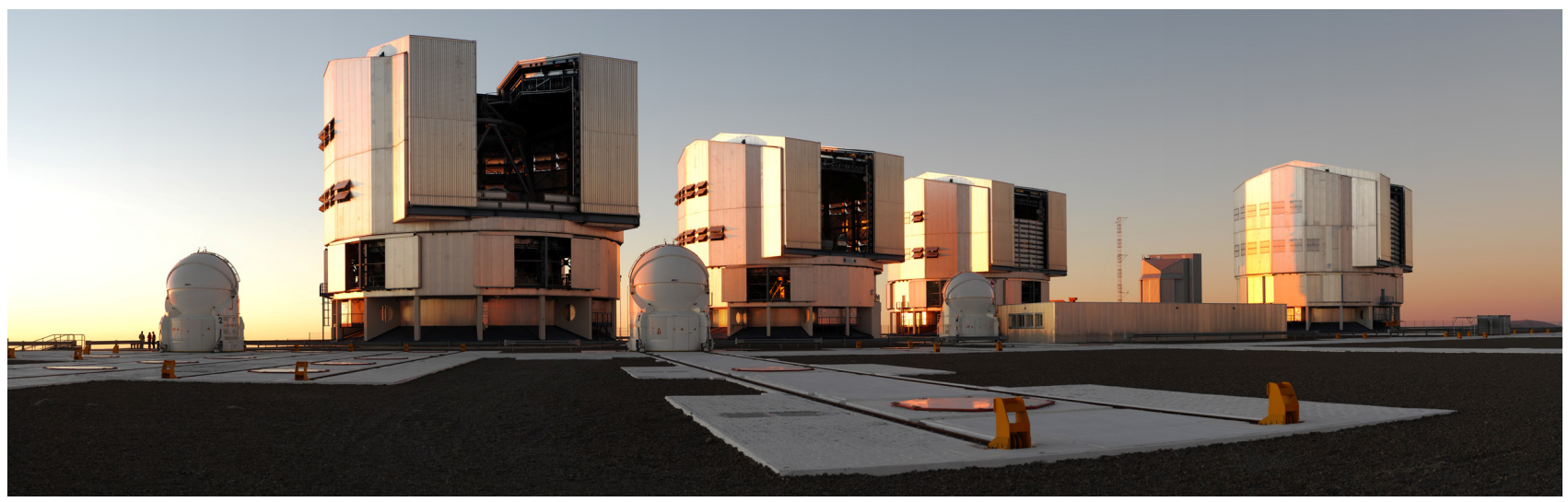

Figure 1: ESO Very Large Telescope on Cerro Paranal (Photo: ESO/Fred Kamphues).

Optomechanics 2011: Innovations and Solutions, edited by Alson E. Hatheway,

Proc. of SPIE Vol. 8125, 812503 - (c) 2011 SPIE · CCC code: 0277-786X/11/\$18

doi: $10.1117 / 12.893682$

Proc. of SPIE Vol. $8125812503-1$ 

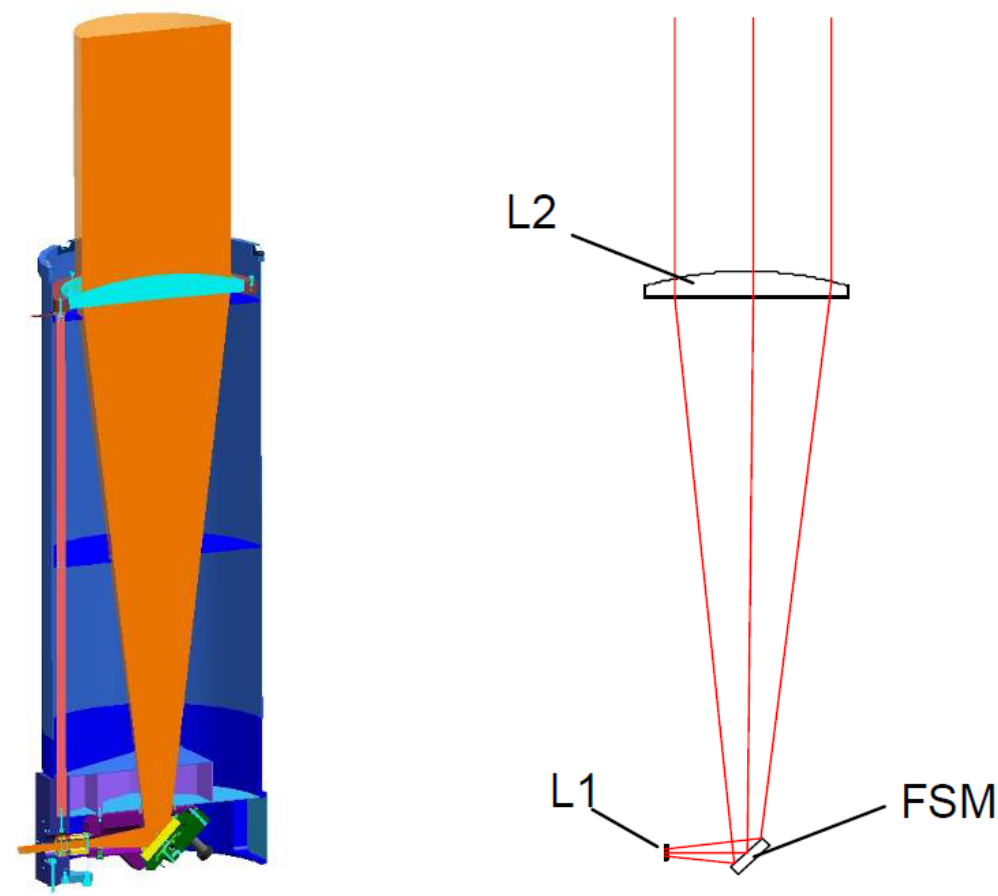

Figure 2: Left: Cross-sectional CAD drawing of the OTA. Right: Code V optical model of the OTA. The FSM is located between the two lenses $(\mathrm{L} 1=\varnothing 30 \mathrm{~mm}$ and $\mathrm{L} 2=\varnothing 380 \mathrm{~mm})$ of a Galilean beam expander.

\section{FIELD SELECTOR MECHANISM DESIGN DESCRIPTION}

The FSM consists of a $\varnothing 100 \mathrm{~mm}$ mirror, which can be rotated around two perpendicular axes in a plane parallel to the mirror surface. Rotation of the FSM mirror results in a reduced and asymmetrical response in the on-sky angle of the laser beam: a factor 0.23 in tip and 0.32 in tilt direction (see Figure 3). The mirror is elastically hinged, and is actuated by high stiffness self-locking actuators. To obtain the required absolute accuracy, contactless sensors are included to directly measure the mirror orientation with respect to the base.

\subsection{Field Selector Mechanism mechanical design}

The Zerodur mirror is bonded to an Invar mirror support, which is constrained in four degrees of freedom (DOF) by a membrane spring and central strut. The membrane spring secures the three DOF in the plane of the mirror, while the central strut secures the single DOF normal to the mirror surface. The remaining two DOF, the tip and tilt directions, are connected to the base through two actuators, which are located at the edge of the mirror support and are spaced $90^{\circ}$ apart around the mirror normal.

The design of the actuators is driven by the dynamic range requirement; few commercial off the shelf actuators can be found with self locking ability, that meet this requirement. TNO has therefore designed a custom actuator, based on a standard Maxon spindle drive (Maxon EC20-flat with 1:53 gear ratio and GP22 S ball screw spindle drive) and a high precision stiffness based transmission. The working principle can be explained from Figure 4: when rotating the motor, the nut compresses a soft spring. The soft spring exerts a force against a high stiffness spring, which is connected to the mirror support. As the stiffness ratio between the soft and stiff spring is 1:22, motion of the nut results in a factor 22 smaller motion of the mirror. This principle increases the resolution dramatically, while compromising only slightly in the dynamic behavior of the FSM; the first Eigen frequency of the stiff spring is $135 \mathrm{~Hz}$. The individual parts of the actuator are shown in Figure 5. 


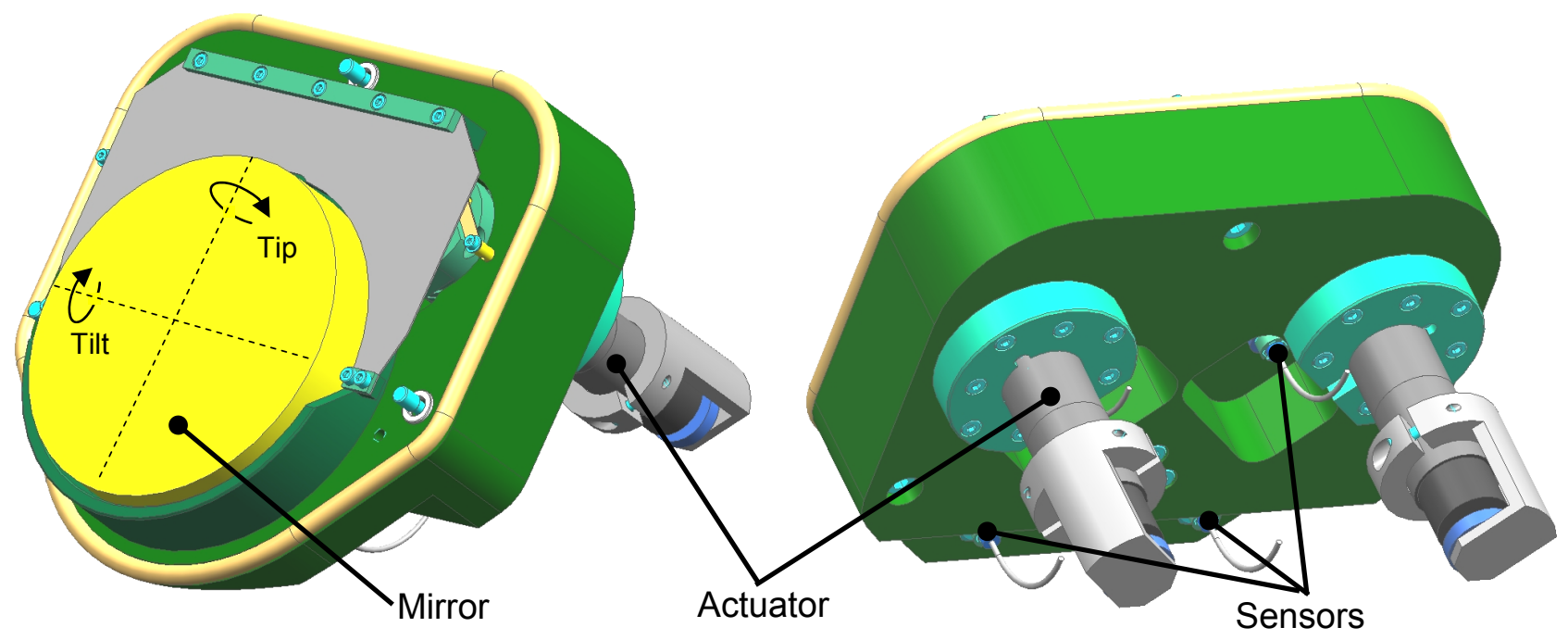

Figure 3: 3D CAD drawing of the Field Selector Mechanism.

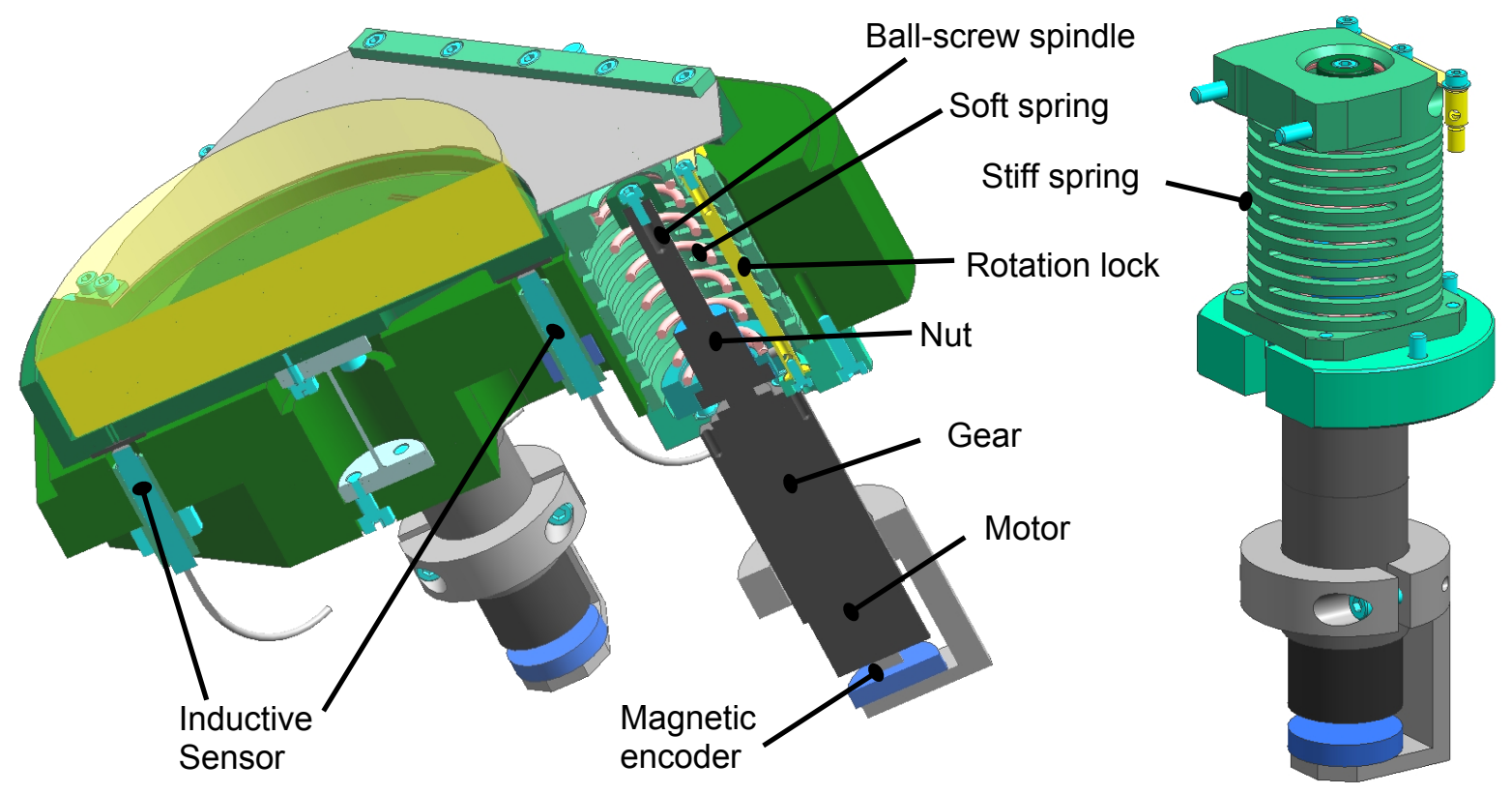

Figure 4: 3D CAD cut-out of the Field Selector Mechanism, showing left: the inside of the FSM and the custom acutator exerting force on the mirror support and right: the custom actuator singled out. 


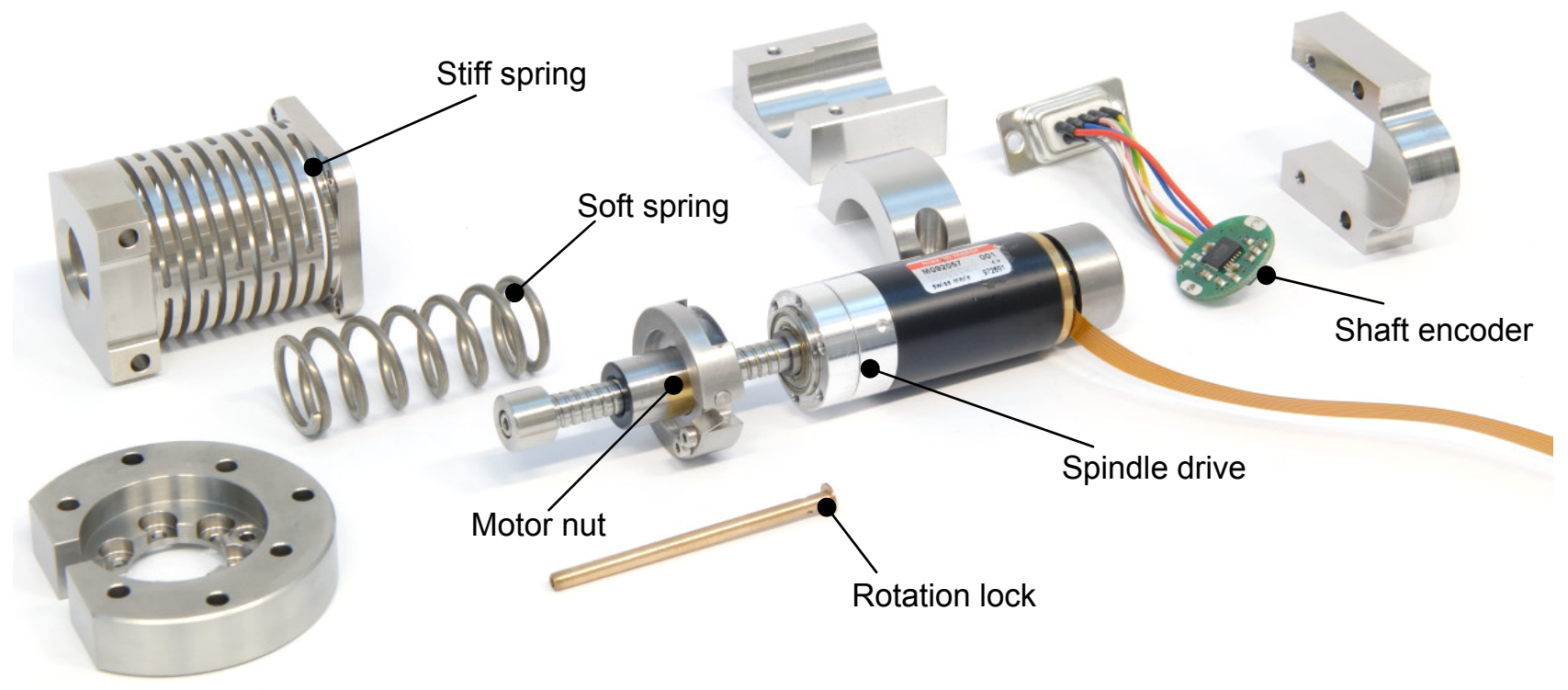

Figure 5: Parts of the Field Selector Mechanism actuator, including a stiffness transmission to increase resolution.

\subsection{Field Selector Mechanism control design}

Besides encoders on the shaft of the Maxon motors (Renishaw RMB20IC), the orientation of the mirror support is measured directly by two pairs of differential inductive position sensors (KAMAN DIT-5200). The two pairs of probes are located at equal arm length from the two actuated axes of rotation, and are mostly insensitive to piston motion. The Maxon motors are driven by ELMO Whistle amplifiers, and the motion control is realized with a Galil RIO Pocket PLC, type 47200. All control electronics are integrated in a small aluminum electronics box, realized by Eltromat BV.

The high pre-stress exerted axially on the motor nut results in significant Coulomb friction in the motor, which varies in magnitude throughout the range. To achieve the absolute accuracy and the required step response dynamics while using only feedback from the inductive sensors, a very high control bandwidth would be necessary. This is undesired, as it would introduce too much noise from the inductive sensors into the control loop. Instead, a local velocity control loop between the actuator and encoder is used to overcome friction and improve the dynamic response of the actuator. Because of the high transmission ratio of the gear box and the springs, the transfer of noise from the encoders to the mirror support is extremely reduced. Based on feedback from the inductive sensors, a much slower cascaded loop is subsequently added to achieve the required accuracy. The velocity loop is implemented in the ELMO Whistle amplifier, while the position loop is implemented on the RIO (see Figure 6).

The PID controller of the velocity loops is auto-tuned to a control bandwidth of approximately $50 \mathrm{~Hz}$. The cascaded position controller consists of only a proportional gain, resulting in a control bandwidth of $5 \mathrm{~Hz}$. To achieve the settling time requirement, velocity feed forward is added to the position loop. 


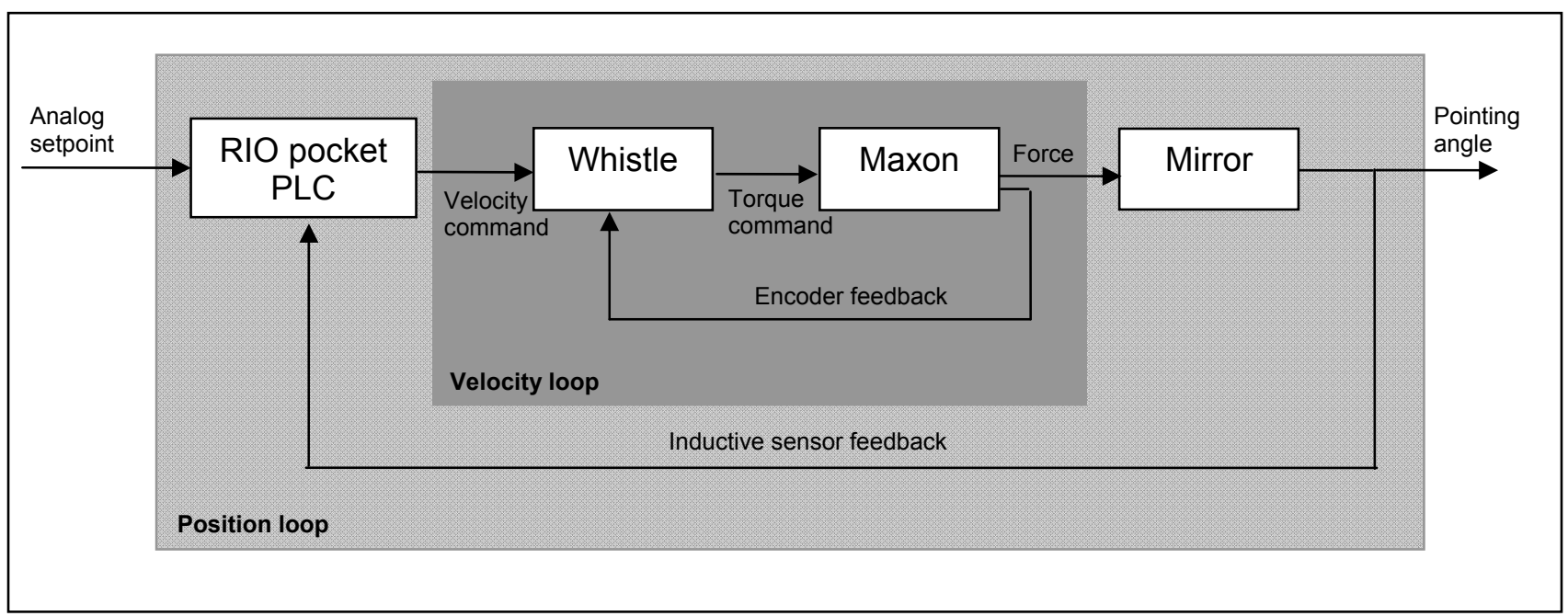

Figure 6: Schematic overview of the control system for the FSM. A local velocity loop is cascaded with the overall inductive sensor feedback loop.

\section{PERFORMANCE TESTING OF THE FIELD SELECTOR MECHANISM}

A first prototype of the FSM is realized in hardware (see Figure 7). The performance of the FSM is tested by measuring the pointing jitter and settling time with an autocollimator. Temperature sensitivity and calibration tests are also performed, but are not reported here.

\subsection{Performance test set-up description}

The FSM is mounted to an optical bread board table, such that the mirror surface is perpendicular to the table surface, when the FSM is in nominal position. The optical bread board table is suspended on air pots, thus providing some isolation from ground vibrations. All control electronics are connected, and the system is operated fully in closed loop, unless reported otherwise. Facing the FSM, an auto-collimator (Newport LDS-Vector) is directed at the center of the mirror. The specifications of the autocollimator are presented in Table 1. The distance between the mirror surface and the autocollimator is approximately $20 \mathrm{~mm}$. Step commands are provided to the FSM electronics through two analog reference inputs, generated with a dSpace 12 bits DA converter.

Table 1: Specifications for the Newport LDS-Vector autocollimator, used in the FSM performance testing.

\begin{tabular}{|l|l|l|}
\hline Specification & Value & Unit \\
\hline Resolution & 0.1 & $\mu \mathrm{rad}$ \\
\hline Beam diameter & 31 & $\mathrm{~mm}$ \\
\hline Range & \pm 2000 & $\mu \mathrm{rad}$ \\
\hline
\end{tabular}




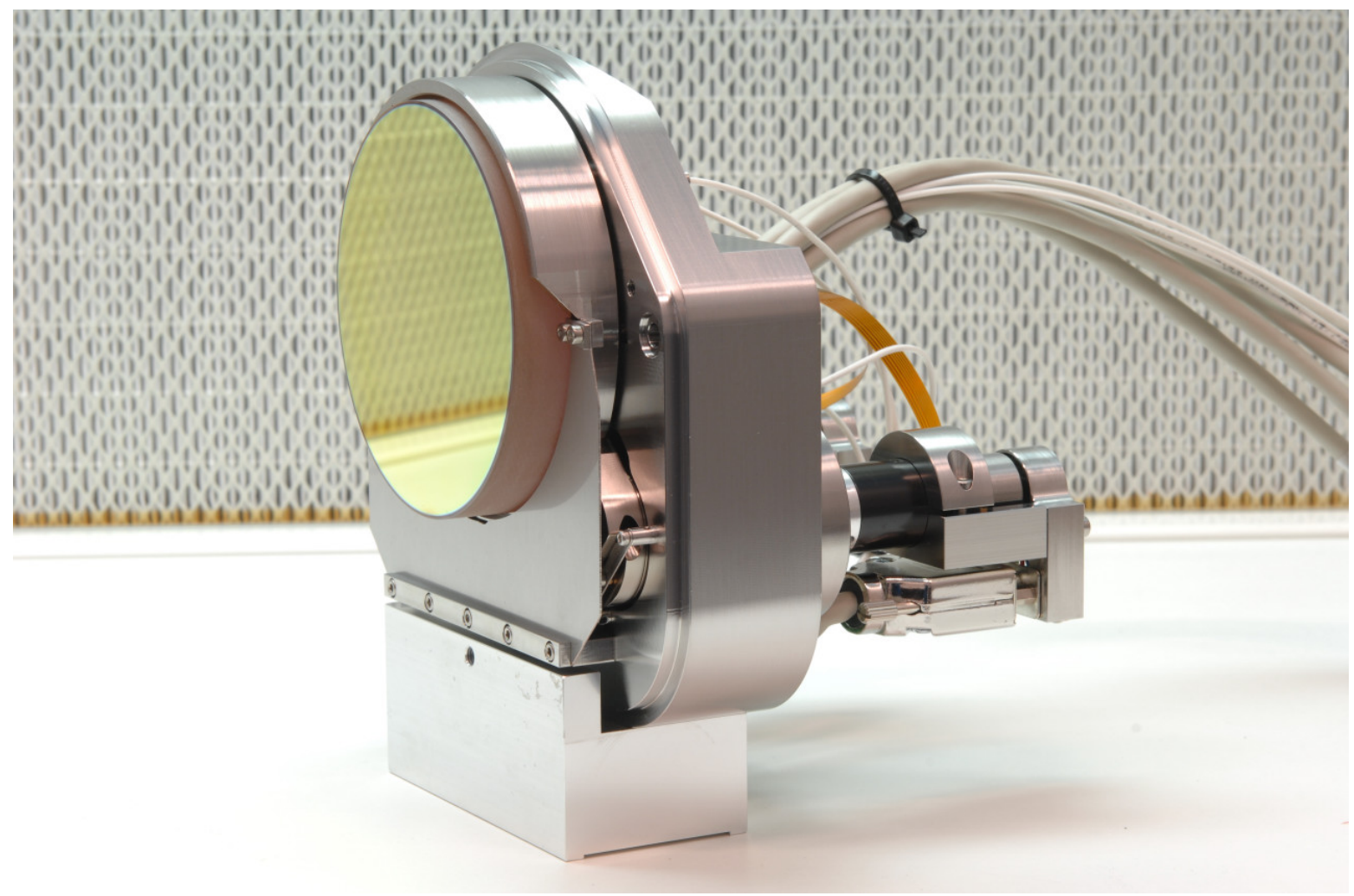

Figure 7: Picture of the first FSM prototype standing upright on a lab table.

\subsection{Performance test description}

The pointing jitter and step response measurements are performed four orientations, as shown in Table 2. The orientations are chosen such that, besides the nominal orientation, the worst case performance can be measured.

Table 2: Orientations of the FSM in which the pointing jitter and step response were measured.

\begin{tabular}{|l|l|l|l|}
\hline $\mathbf{R x}$ & $\mathbf{R y}$ & Unit & Configuration \\
\hline 0 & 0 & $\mathrm{mrad}$ & Both motors in nominal position \\
\hline 4.3 & 4.3 & $\mathrm{mrad}$ & $\begin{array}{l}\text { One motor in nominal position, } \\
\text { one motor in extreme position }\end{array}$ \\
\hline 0 & 6.1 & $\mathrm{mrad}$ & $\begin{array}{l}\text { Both motors in same extreme } \\
\text { position }\end{array}$ \\
\hline 6.1 & 0 & $\mathrm{mrad}$ & $\begin{array}{l}\text { Both motors in opposite extreme } \\
\text { position }\end{array}$ \\
\hline
\end{tabular}

The pointing jitter is measured with the autocollimator in the tip and tilt direction of the FSM, for each orientation as described in Table 2. Measurements were performed with $500 \mathrm{~Hz}$ sampling frequency, and a length of 20 seconds. The performance measurements are done with the set point input short circuited, as the noise level of the analog set point signal is not specified. From the time data, the Amplitude Spectral Density (ASD) is calculated by averaging with a Hanning window of $1 / 5$ of the length of the time sequence, and with $50 \%$ overlap. Integration of the Amplitude Spectral 
Density is downward, resulting in the Cumulative Spectral Density. Each value represents the RMS value $(1 \sigma)$ of the signal above that frequency. After evaluating the results, an additional measurement of the pointing jitter at nominal orientation was performed, this time with a sampling frequency of $5 \mathrm{~Hz}$ and a measurement length of 2000 seconds.

Step responses are achieved by putting a step on the set-point voltage, first in tip $(21.2 \mu \mathrm{rad})$ and then in tilt $(15.0 \mu \mathrm{rad})$ direction, and both up and down, for each orientation as listed in Table 2. Note that these tests were performed before calibration, and the magnitude of the measured step can be slightly off. The step command is synchronized with the autocollimator measurement, to include delay in the FSM response.

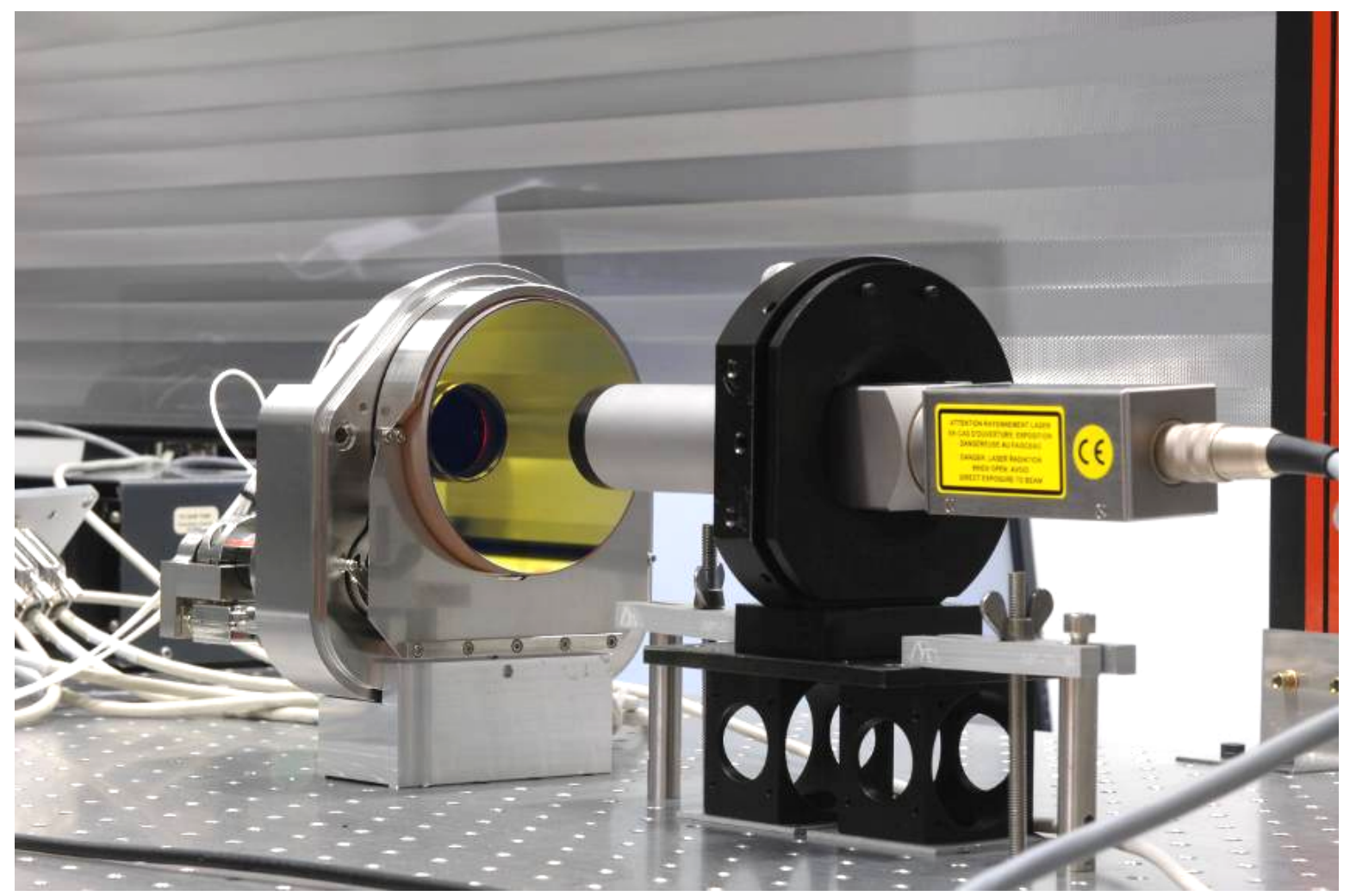

Figure 8: Picture of the FSM performance test set-up. An autocollimator is facing the FSM mirror, to accurately measure the performance in tip and tilt direction.

\subsection{Pointing jitter test results}

The closed loop pointing jitter measurement results for two FSM mirror orientations are shown in Figure 9. Additionally, the nominal position is measured in open loop. Left: Amplitude spectral density results for tip (above) and tilt (below) directions. Right: Cumulative spectral density results for tip (above) and tilt (below) directions. The amplitude spectral densities show that the closed loop system introduces noise below $5 \mathrm{~Hz}$ only. The cumulative spectral density, showing the root-mean-square of pointing jitter above each frequency, is less than $0.4 \mu \mathrm{rad}$ RMS for all orientations measured.

The cumulative spectral densities show a significant increase towards low frequencies. An additional closed loop performance measurement is performed to show the behaviour down to $0.003 \mathrm{~Hz}$, at nominal orientation. The results are shown in Figure 10. 

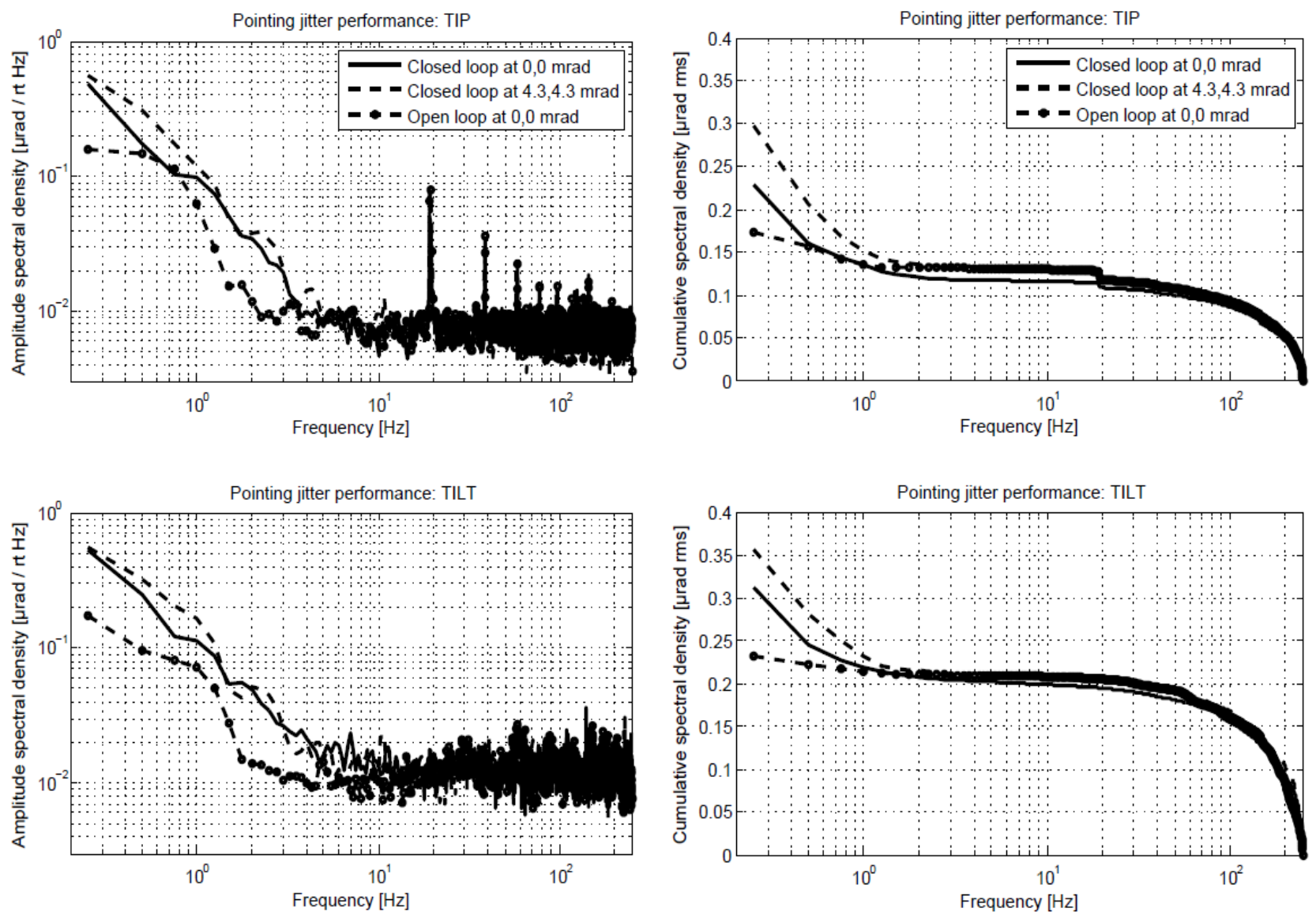

Figure 9: Pointing jitter performance results of the FSM, in tip and tilt direction, as measured by the autocollimator. The figure shows the closed loop performance for two orientations: $0,0 \mathrm{mrad}$ and 4.3, $4.3 \mathrm{mrad}$, as well as the open loop performance for $0,0 \mathrm{mrad}$.
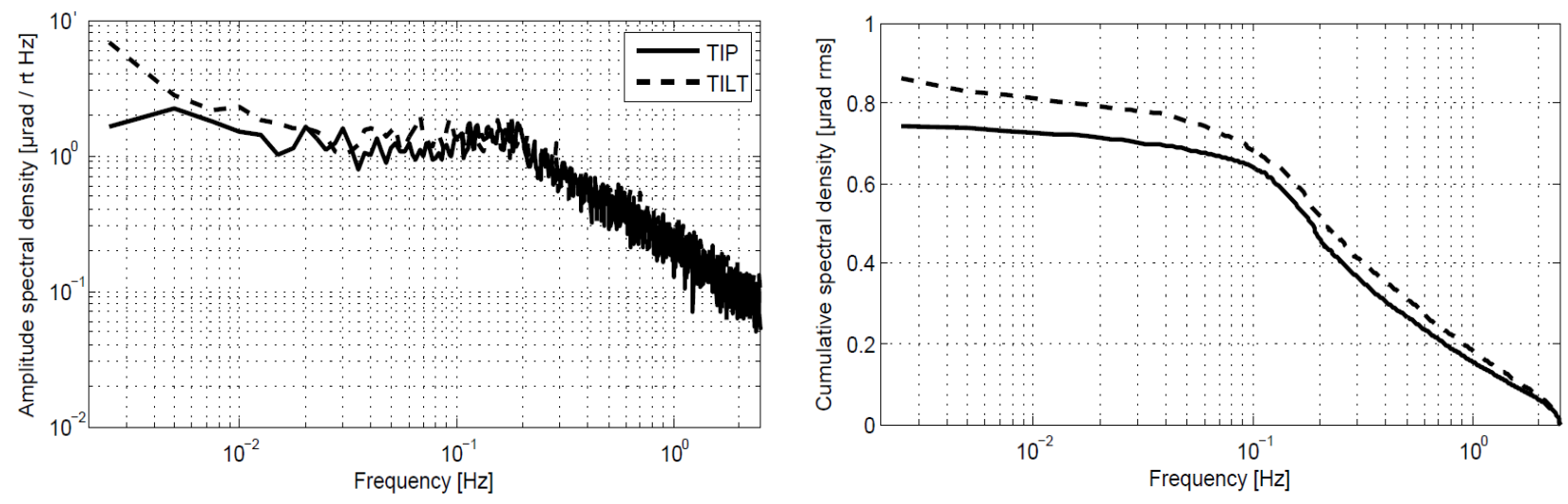

Figure 10: Long pointing jitter measurement to show the behaviour of the FSM for low frequencies. Left: Amplitude spectral density measured at 0,0 mrad. Right: Cumulative spectral density measured at 0,0 mrad. 
The step response results for the same two FSM mirror orientations are shown in Figure 11. Note that these step responses are performed before calibration of the differential inductive sensors; a slight deviation from the intended value is therefore visible in the results.
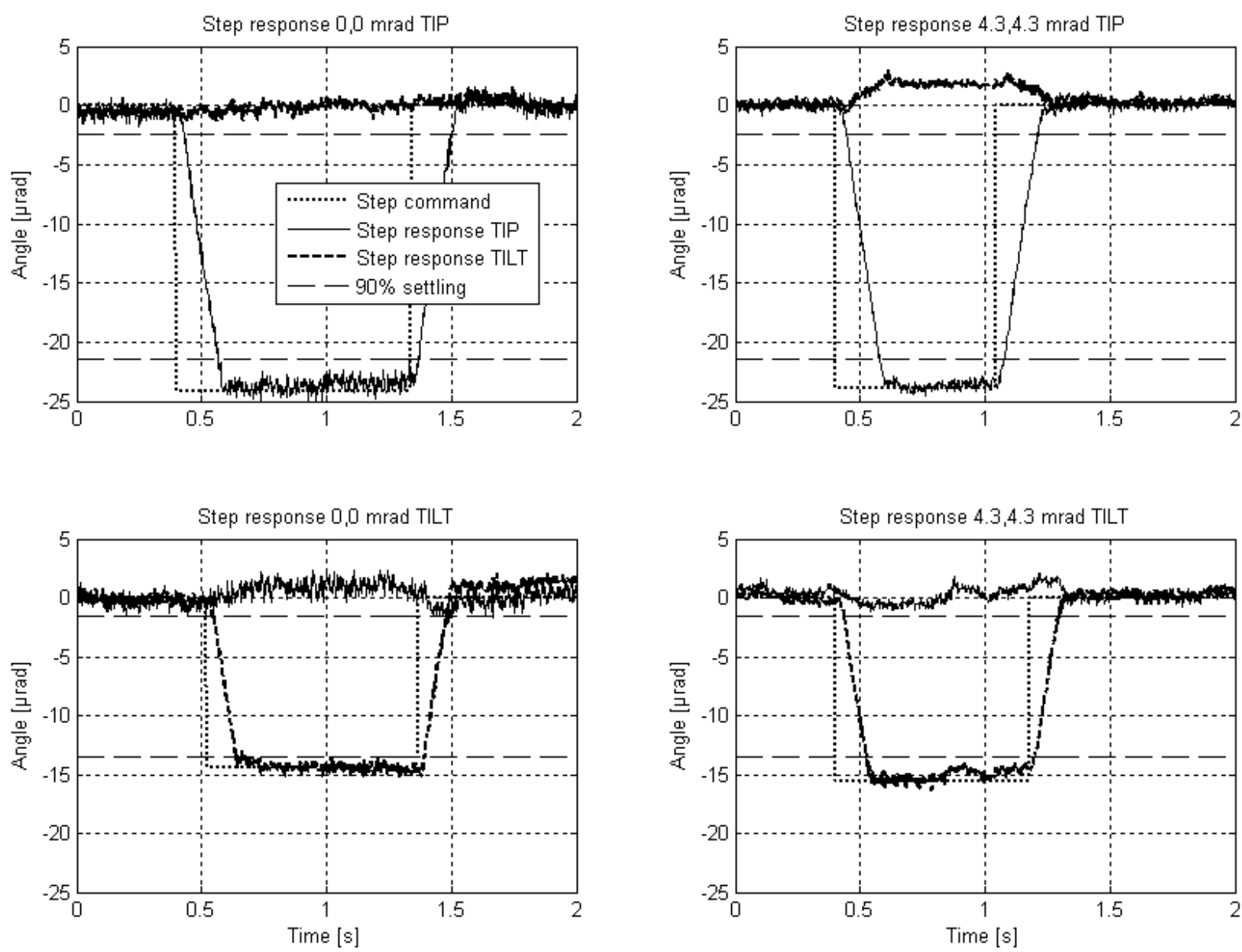

Figure 11: Step responses of the FSM as measured by the autocollimator. The figure shows responses for two orientations: $0,0 \mathrm{mrad}$ and 4.3, $4.3 \mathrm{mrad}$.

\section{CONCLUSIONS}

The VLT Laser Guide Star facility requires a stable beam expander with a tip/tilt mirror to cover a large on-sky field of view. The Optical Tube Assembly, containing the Field Selector Mechanism, has been designed, realized and successfully tested by TNO. The design of the FSM is based on a Zerodur mirror, bonded to a membrane spring - strut combination to allow only tip/tilt motion. Actuation is realized with a custom spindle drive actuation and a stiffness based transmission. Differential inductive sensor pairs provide absolute accuracy of the mirror orientation. To overcome friction in the spindle drive, a local velocity control loop is cascaded with the overall low bandwidth feedback loop.

The amplitude spectral densities of the pointing jitter of the FSM mirror, as measured with an autocollimator, show that the jitter of the mirror is only increased below the inductive sensor feedback loop, as can be expected. Integration of these spectra shows that the RMS of the pointing jitter down to $0.2 \mathrm{~Hz}$ is below $0.4 \mu \mathrm{rad}$ rms. An additional measurement is performed to show the behaviour of the pointing jitter towards lower frequencies. Figure 10 shows that the jitter increases down to $0.2 \mathrm{~Hz}$, and then flattens out. At $0.003 \mathrm{~Hz}$, the RMS pointing jitter is $1 \mu \mathrm{rad}$ RMS and thus well within the requirement of $1.5 \mu \mathrm{rad}$ rms. Together with temperature sensitivity and calibration test results, which are 
not reported in this paper, the absolute accuracy requirement is met. All step responses are within 0.2 seconds. The results show a slight variation in step size as well as some cross-coupling, caused by the fact that the step responses were performed before calibration of the FSM. On top of the step response signal, the pointing jitter is also visible in the results.

The strict performance requirements on the FSM are shown to be met, and the FSM is ready to be implemented in the first Optical Tube Assembly for further unit level testing.

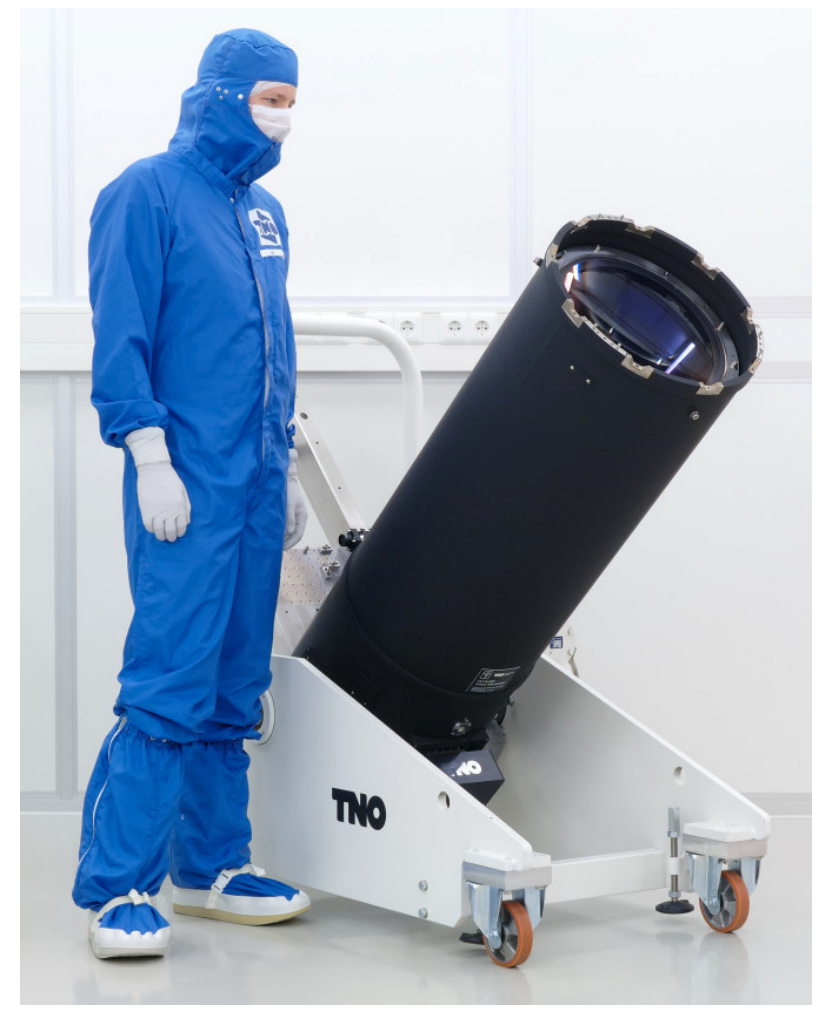

Figure 12: The first Optical Tube Assembly containing the Field Selector Mechanism,as mounted in its testing rig.

\section{ACKNOWLEDGEMENTS}

TNO would like to thank ESO for awarding the contract for the realization of the Optical Tube Assemblies for the Very Large Telescope. Furthermore, the realization of the Field Selector Mechanism would not have been possible without the help and support of Eltromat BV, Maxon Motors BV, GL Precision BV, Vacutech BV and Rocky Mountain Instrument Co..

\section{REFERENCES}

[1] ESO Garching, [The VLT White Book] (1998).

[2] Arsenault, R., et al., "Progress on the VLT Adaptive Optics Facility", ESO Messenger No. 142 (2010).

[3] Fugate, R. Q., Fried, D. L., Ameer, G. A., Boeke, B. R., Browne, S. L., Roberts, P. H., Ruane, R. E., Tyler, G. A. and Wopat, L. M., "Measurements of atmospheric wavefront distortion using scattered light from a laser guide-star," Nature 353, 144-146 (1991). 